\section{Literature Cited}

1) Bloomfield, V. A. and R. K. Dewan: J. Phys. Chem., 75, 3113 (1971).

2) Bearman, R. J. and J. G. Kirkwood: J. Chem. Phys., 28, 136 (1958).

3) Bearman, R. J. and P. E. Jones: ibid., 33, 1432 (1960).

4) Cohen, M. H. and D. Turnbull: ibid., 31, 1164 (1959).

5) Grasstone, S., K. T. Laidler and H. Eyring: "The Theory of Rate Processes," McGraw Hill, New York (1941).

6) Green, H. S.: "The Molecular Theory of Fluids", North Holland, Amsterdam (1952).

7) Guggenheim, E. A.: Proc. Roy. Soc., A148, 305 (1935).

8) Harada, M., M. Tanigaki, A. Nishimura and W. Eguchi: J. Chem. Eng. Japan, 7, 407 (1974).

9) Heric, E. L. and J. G. Brewer: J. Chem. Eng. Data, 12, 574
(1967).

10) Ishikawa, T.: “Kongo Eki Nendo no Riron," Maruzen, Tokyo (1968).

11) Macedo, P. B. and T. A. Litovitz: J. Chem. Phys., 42, 245 (1965).

12) McAllister, R.: AIChE J. 4., 6, 427 (1960).

13) McLaughlin, I. L. and H. T. Davis: J. Chem. Phys., 45, 2020 (1966).

14) Landolt-Börnstein: “Zahlenwerte und Funktionen," II, Teil 5, Springer-Verlag (1969).

15) Rice, S. A. and A.R. Allnatt: J. Chem. Phys., 34, 409 (1961).

16) Ridgway, K. and P. A. Batler: J. Chem. Eng. Data, 12, 509 (1967).

17) Saraiya, N. K. and J. Winnick: J. Chem. Phys., 49, 1447 (1968).

\title{
A GENERAL CORRELATION FOR NON-POLAR GAS SOLUBILITIES IN AQUEOUS ALCOHOL SOLUTIONS
}

\author{
JUNJI TOKUNAGA \\ Department of Chemical Engineering, Kansai University, Suita, 560
}

\begin{abstract}
From the solubility data of oxygen, nitrogen, and carbon dioxide in aqueous solutions of methanol, ethanol, 1-propanol, and 2-propanol, a general correlation is obtained by plotting the excess quantities for the Ostwald coefficients averaged by volume fraction against the solution composition.

The relation between the excess quantities in aqueous solutions of an alcohol and the alcohol volume fraction in the mixed solvents is given by a curve regardless of the kind of solute gas. The relation is shown as a curve for each kind of alcohol in the solutions.

Qualitative similarity between the excess quantity and the ratio of the excess molar volume to the volume on ideal mixing for each aqueous alcohol solution is recognized and discussed.
\end{abstract}

\section{Introduction}

The estimation of gas solubilities in mixed solvents is an important problem for engineering purposes. Most of the previous studies ${ }^{3-10}$ concerning gas solubilities in mixed solvents have been confined mainly to non-aqueous liquids, and few reports for aqueous alcohol solvents have been presented.

The author et al. measured the solubilities of $\operatorname{oxygen}^{11)}$, nitrogen ${ }^{11}$, and carbon dioxide ${ }^{11,12)}$ in aqueous solutions with methanol, ethanol, 1-propanol, or 2-propanol.

As aqueous solutions are highly non-ideal liquids, theoretical interpretation for gas solubilities in these solutions is not expected at present. For obtaining a general correlation of non-polar gas solubilities in aqueous alcohol solutions, therefore, these solubility data $^{11,12)}$ have been analyzed by use of the excess

Received June 3, 1974. quantity of gas solubility in mixed solvent.

This paper is concerned with correlations for nonpolar gas solubilities in aqueous alcohol solvents.

\section{Excess Quantity}

Few definitions for the excess quantity of gas solubility in mixed solvents have been proposed: they are the excess Henry's constant on the basis of mole fraction $^{4,10)}$ or volume fraction ${ }^{2,7)}$, and the excess Ostwald coefficient ${ }^{6)}$ on the basis of volume fractions, as follows:

$$
\begin{aligned}
& \ln H_{\mathrm{R}, \mathrm{mix}}^{\mathrm{E}}=\ln H_{\mathrm{R}, \mathrm{mix}}-\sum_{i=1}^{2} x_{i} \ln H_{\mathrm{R}, i} \\
& \ln \kappa=\ln H_{\mathrm{R}, \text { mix }}-\sum_{i=1}^{2} \Phi_{i} \ln H_{\mathrm{R}, i} \\
& \ln \kappa(L)=\ln L_{\mathrm{R}, \text { mix }}-\sum_{i=1}^{2} \Phi_{i} \ln L_{\mathrm{R}, i}
\end{aligned}
$$

The excess quantity by Eq. (1), $\ln H_{\mathrm{R}, \text { mix }}^{\mathrm{E}}$ was first shown by Krichevsky ${ }^{4}$. O'Connell and Praus- 


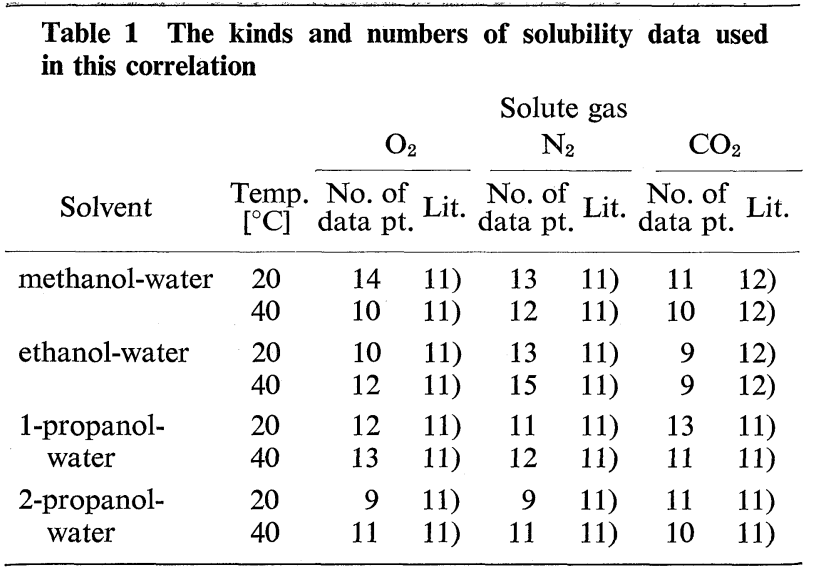

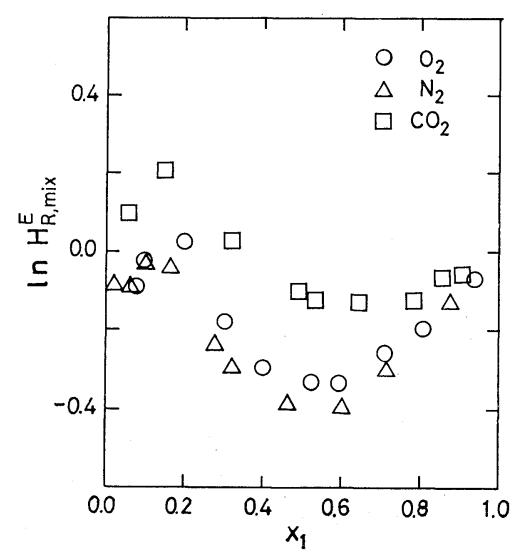

Fig. 1 Excess Henry's constants of oxygen, nitrogen, and carbon dioxide in ethanol(1)-water(2) solutions at $20^{\circ} \mathrm{C}$

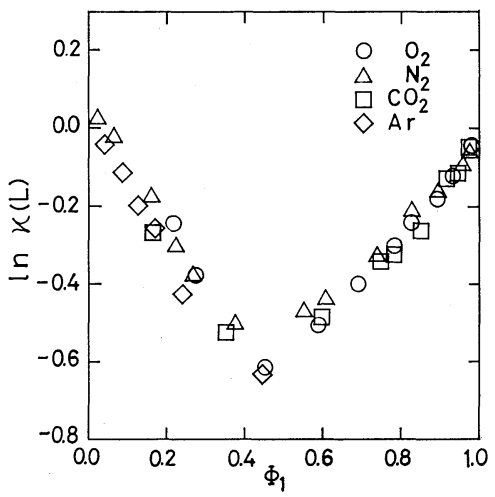

Fig. 2 Excess quantities, $\ln \kappa(L)$, of oxygen, nitrogen, and carbon dioxide in ethanol (1)-water (2) solutions at $20^{\circ} \mathrm{C}$

nitz ${ }^{9}$ have shown $\ln H_{R}^{\mathrm{E}}$, mix in terms of one Margules constant for a gas-free solvent mixture.

The theoretical meanings and the interrelation of $\ln \kappa$ and $\ln \kappa(L)$, defined by Eqs. (2) and (3) respectively, were discussed by Nitta and Katayama ${ }^{6}$. They also showed that $\ln \kappa$ and $\ln \kappa(L)$ are superior to $\ln H_{\mathrm{R}, \mathrm{mix}}^{\mathrm{E}}$ for theoretical analysis of the gas solubility in mixed solvents.

In aqueous alcohol solutions, both components are polar and self-associated liquids. Water molecules constitute ice-like structures and alcohol molecules form linear association preferentially. In addition, association between unlike molecules is also seen in the solutions. As no satisfactory explanation for such complicated solutions is available, the observed excess quantities, $\ln \kappa$ or $\ln \kappa(L)$, for gas solubilities in aqueous alcohol solutions could not be correlated by means of the thermodynamic properties of the solvent components.

However, some empirical correlations were obtained by plotting the excess quantities, $\ln \kappa(L)$, against the alcohol volume fraction for aqueous alcohol solutions.

The plots of $\ln \kappa$ vs. the alcohol volume fraction in aqueous alcohol solutions also gave a similar correlation.

However, the excess quantity, $\ln \kappa(L)$, was used for the correlation in this work, because the expression of Ostwald coefficient is favorable to an understanding of the relation between $\ln \kappa(L)$ and the excess molar volume, which is described in a later section.

Table 1 shows the kinds and numbers of solubility data in this correlation.

\section{Correlations and Discussion}

\section{Effect of the kind of solute gas on excess quantities}

Fig. 1 shows the relations between the excess Henry's constants, $\ln H_{\mathrm{R}}^{\mathrm{E}}$ mix , at $20^{\circ} \mathrm{C}$ for the systems of nitrogen, oxygen, and carbon dioxide in aqueous ethanol solutions. A separate curve is obtained for each solute gas in this expression.

The plots of the excess quantity, $\ln \kappa(L)$, at $20^{\circ} \mathrm{C}$ against alcohol volume fraction for the same data are given in Fig. 2. By use of the excess quantity, $\ln \kappa(L)$, all observed values for the aqueous ethanol solutions conform to one curve regardless of the kind of solute gas.

The excess quantity, $\ln \kappa(L)$, for aqueous ethanol solution at $40^{\circ} \mathrm{C}$ was also independent of the kind of solute gas as the case at $20^{\circ} \mathrm{C}$, but the degree of deviation from ideality at $40^{\circ} \mathrm{C}$ was smaller than that at $20^{\circ} \mathrm{C}$.

The solubility data for argon in aqueous ethanol solutions by Ben-Naim and Baer ${ }^{1)}$, which are available only for low alcohol concentrations, are also plotted in Fig. 2. These plots are close to the curve given by other solute gases.

In aqueous solutions of other alcohols, the differences in the excess values, $\ln \kappa(L)$, owing to the kind of solute gas were comparatively small, as in aqueous ethanol solutions.

\section{Effect of the kind of solvent on excess quantities}

For aqueous solutions with methanol, ethanol, 1-propanol, or 2-propanol, the relation between the excess quantities, $\ln \kappa(L)$, and the alcohol volume fraction in the mixed solvents was examined. The 


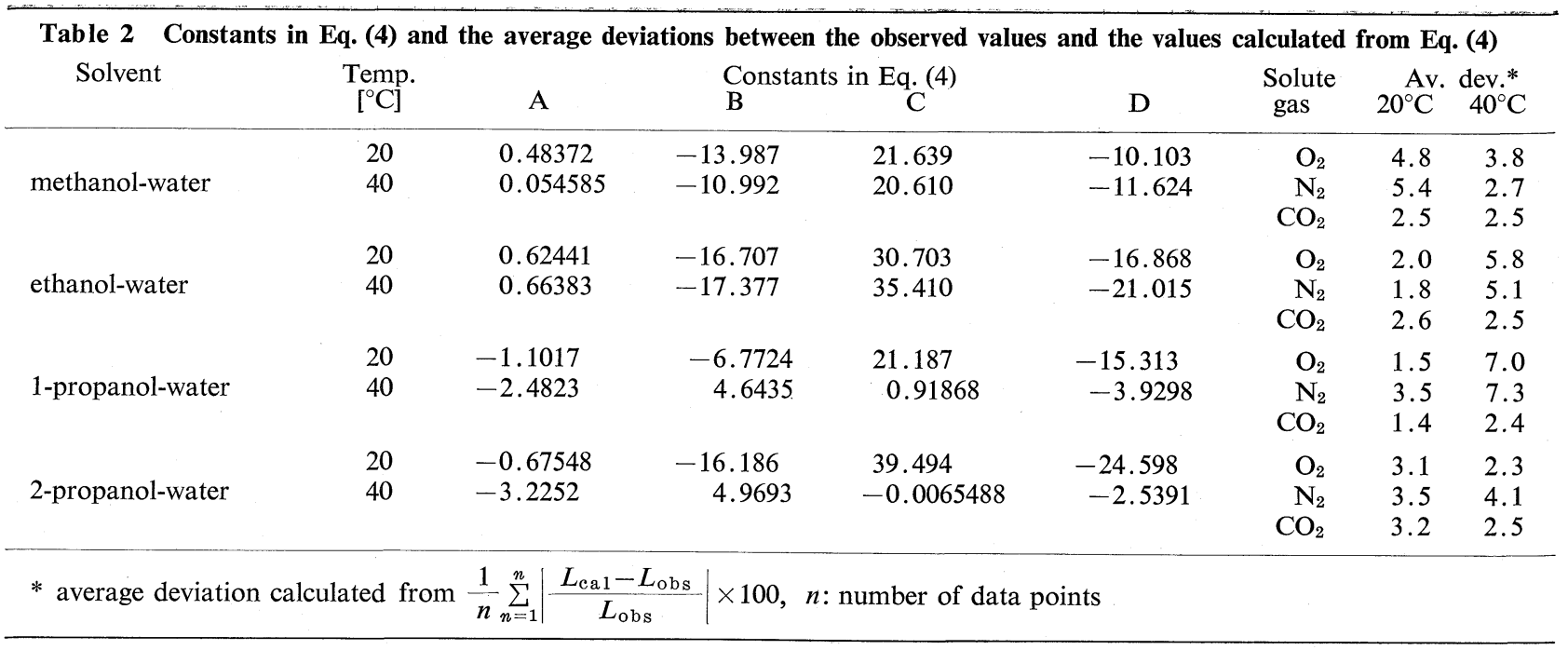

results for these aqueous alcohol solutions are obtained as parabolic curves, different for each alcohol solution.

Whether the excess values for each solution can be fitted to the following polynomial equation of RedlichKister type was examined.

$$
\ln \kappa(L)=\Phi_{1} \Phi_{2}\left(\mathrm{~A}+\mathrm{B} \Phi_{1}+\mathrm{C} \Phi_{1}^{2}+\mathrm{D} \Phi_{1}^{3}\right)
$$

Constants in Eq. (4) for four aqueous alcohol solutions were determined by the least-square procedure from the solubility data of nitrogen, oxygen, and carbon dioxide. Table 2 shows the constants in Eq. (4) for aqueous solutions of four alcohols and the average deviations between the excess quantities observed and those calculated from Eq. (4).

The curves have minima at about 0.5 of alcohol volume fraction, and the values of $\ln \kappa(L)$ decrease in the order: 1-propanol $>2$-propanol $>$ ethanol $>$ methanol. Fig. 3 shows the curves calculated from Eq. (4) using the constants given in Table 2 against the volume fraction of alcohol in aqueous alcohol solution.

\section{Comparison of excess quantities $\ln \kappa(L)$ with excess molar volume}

When alcohol and water are mixed, contraction in volume occurs. If the solute gas dissolves in the free volume of the solvents, the contraction of volume in the mixed solvents diminishes the space for the dissolving gas. Fig. 4 shows the relation between the ratio of the excess molar volume to the volume of ideal mixture, $v^{\mathrm{E}} / v^{\circ}$, and the alcohol volume fraction in aqueous alcohol solutions.

$$
\left.\begin{array}{l}
v^{\mathrm{E}}=v-\sum_{i=1}^{2} x_{i} v_{i} \\
v^{\mathrm{E}} / v^{\circ}=v^{\mathrm{E}} / \sum_{i=1}^{2} x_{i} v_{i}
\end{array}\right\}
$$

The quantity of $v^{\mathrm{E}} / v^{\circ}$ was calculated from the density data for aqueous alcohol solutions at $20^{\circ} \mathrm{C}$. In comparison of the curves of excess quantities in

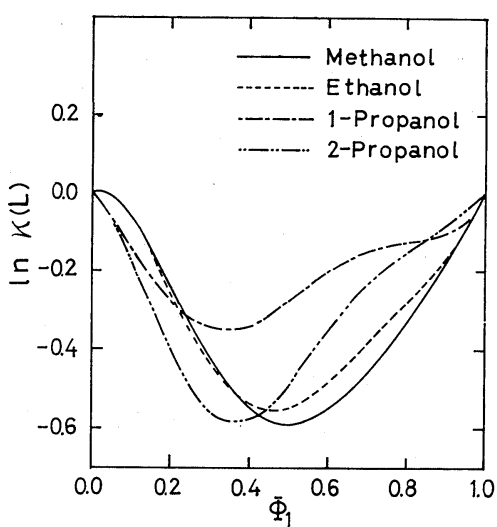

Fig. 3 Excess quantities, $\ln \kappa(L)$, calculated from Eq. (4) for slightly soluble gases in methanol (1)-water (2), ethanol (1)-water (2), 1-propanol(1)-water(2), and 2-propanol(1)water (2) solutions at $20^{\circ} \mathrm{C}$

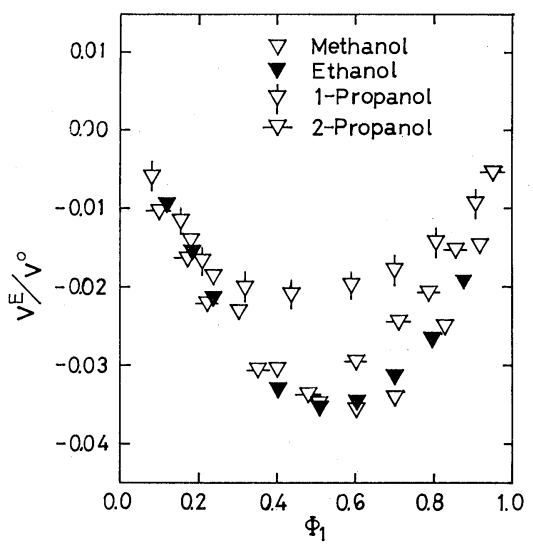

Fig. 4 The plots of $v^{\mathrm{E}} / \boldsymbol{v}^{\circ}$ values against $\Phi_{1}$ for aqueous solution of each alcohol at $20^{\circ} \mathrm{C}$

Fig. 3 with the curves of $v^{\mathrm{E}} / v^{\circ}$ in Fig. 4, similarities are recognized in shape and in alcohol concentrations showing minimum excess quantities. It is difficult to correlate these two values quantitatively, but the tendency of $v^{\mathrm{E}} / v^{\circ}$ may be useful for predicting qualitatively the excess quantity $\ln \kappa(L)$ for non-polar gases in aqueous alcohol solutions. 
The similarity between $\ln \kappa(L)$ in aqueous solutions of four alcohols and $v^{\mathrm{E}} / v^{\circ}$ in gas-free mixtures is considered as evidence for the fact that the excess values of solute gases in each aqueous alcohol solution, $\ln \kappa(L)$, are mainly subject to the kind of solvent and independent of the kind of solute gas.

\section{Conclusion}

On the excess quantity $\ln \kappa(L)$ of slightly soluble non-polar gases in aqueous solutions of each alcohol, there is a general correlation which is independent of the kind of gas.

The plots of $\ln \kappa(L)$ for aqueous alcohol solutions of four kinds vs. alcohol composition give different curves for each alcohol. The excess quantity $\ln \kappa(L)$ for aqueous alcohol solutions are expressed by Eq.(4) of the Redlich-Kister type, and the constants in Eq. (4) are tabulated in Table 2.

Qualitative similarity is recognized between the quantity of $v^{\mathrm{E}} / v^{\circ}$ in the solution and $\ln \kappa(L)$ against the alcohol volume fraction in the solutions.

\section{Acknowledgment}

The author wishes to express his thanks to Prof. Takashi Katayama and Mr. Tomoshige Nitta of Osaka University for their valuable suggestions.

\section{Nomenclature}

$$
\begin{array}{ll}
\text { A, B, C, D } & =\text { empirical constants in Eq. (4) } \\
H & =\text { Henry's constant } \\
L & =\text { Ostwald coefficient } \\
v & =\text { molar volume in liquid phase } \\
x & =\text { mole fraction in liquid phase }
\end{array}
$$

$$
\begin{array}{lll}
\kappa & =\text { excess quantity defined in Eq. (2) } & {[-]} \\
\kappa(L) & =\text { excess quantity defined in Eq. (3) } & {[-]} \\
\Phi & =\text { volume fraction in liquid phase } & {[-]}
\end{array}
$$

$\langle$ Subscripts〉
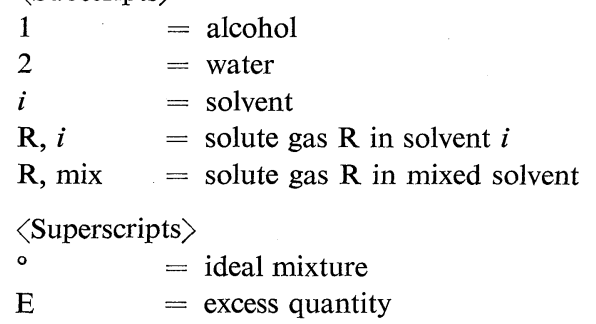

\section{Literature Cited}

1) Ben-Naim, A. and S. Baer: Trans. Faraday Soc., 60, 1736 (1964).

2) Hildebrand, J. H., J. M. Prausnitz and R. L. Scott: "Regular and Related Solutions", Van Nostrand Reinhold, N.Y. (1970).

3) Koudelka, L.: Chem. zvesti, 18, 178; 18, 493 (1964).

4) Krichevsky, I. R.: Zhur. Fiz. Khim., 9, 41 (1937).

5) Leites, I. L. and F. P. Ivanovskii: Khim. Prom., 9, 653 (1962).

6) Nitta, T. and T. Katayama: J. Chem. Eng. Japan, 7, 310 (1974).

7) Nitta, T., A. Tatsuishi, and T. Katayama: ibid., 6, 475 (1973).

8) O’Connell, J. P.: AIChE J., 17, 658 (1971).

9) O'Connell, J. P. and J. M. Prausnitz: Ind. Eng. Chem., Fundamentals, 3, 347 (1964).

10) Tiepel, E. W. and K. E. Gubbins: Can. J. Chem. Eng., 50, 361 (1972).

11) Tokunaga, J.: J. Chem. Eng. Data, 20, 41 (1975).

12) Tokunaga, J., T. Nitta, and T. Katayama: Kagaku Kögaku, 33, 775 (1969). 